\title{
Varón de 61 años con deterioro cognitivo subagudo y ataxia de la marcha
}

\author{
61-year-old male with subacute cognitive impairment and ataxia
}

\author{
Amara González Noya ${ }^{1}$, Ana María Lorenzo Vizcaya', Raquel Fernández González¹, Diego Rodríguez Gómez² \\ ${ }^{1}$ Servicio de Medicina Interna. ${ }^{2}$ Servicio de Neurología. Complexo Hospitalario Universitario de Ourense
}

\begin{abstract}
Resumen
La enfermedad de Creutzfeldt-Jakob (ECJ) forma parte de las enfermedades priónicas. La presentación típica consiste en la presencia de demencia y mioclonías, en pacientes jóvenes; con una evolución rápidamente progresiva y con una elevada mortalidad de forma temprana.

Para el diagnóstico es fundamental el estudio histológico del tejido cerebral; aunque la presentación clínica típica, los estudios de imagen (RMN), el electroencéfalograma y la presencia de proteína 14-3-3 en el estudio de líquido céfalorraquídeo pueden orientar con alta probabilidad al diagnóstico de ECJ. Palabras clave: Creutzfeldt-Jakob. Enfermedades priónicas. Demencia
\end{abstract}

\section{Introducción}

La enfermedad de Creutzfeldt-Jakob (ECJ) forma parte de las enfermedades priónicas. La presentación típica consiste en la presencia de demencia y mioclonías, en pacientes jóvenes; con una evolución rápidamente progresiva y con una elevada mortalidad de forma temprana.

En este artículo, presentamos el caso de un varón de mediana edad que debuta con alteraciones de la marcha y cambios sutiles en funciones mentales superiores, lo que dificultó el diagnóstico final; al tratarse de una causa de demencia de muy baja prevalencia, por lo que no suele ser considerada en el diagnóstico diferencial habitual en pacientes con deterioro cognitivo, y cuyo diagnóstico es de especial importancia por el pobre pronóstico a corto plazo y las complicaciones clínicas que aparecen durante su evolución, que suponen un manejo complicado del paciente y difícil aceptación por parte de las familias.

\section{Caso clínico}

Varón de 61, sin hábitos tóxicos, trabajador en tienda de textiles y con antecedentes de dislipemia a tratamiento con atorvastatina y síndrome ansioso-depresivo de décadas de evolución a tratamiento con bromazepam y clomipramina.

Ingresó en el Servicio de Neurología por alteraciones de la memoria y marcha inestable. La familia refería desde hace tres meses cambios conductuales en el paciente, con tendencia a la apatía, además de pequeños olvidos y pérdida de memoria, presentando en semanas posteriores exacerbación de la clínica, con olvidos cada más frecuentes, además de episodios de desorientación témporoespacial y alteración de la marcha, decidiendo su ingreso.

Al ingreso, presentaba constantes mantenidas y la exploración cardiopulmonar fue normal. En la exploración neurológica destacaba

\begin{abstract}
Creutzfeldt-Jakob disease (CJD) is the most frequent of the human prion diseases, although it is still rare. The typical presentation is the presence of dementia and myoclonus, in young patients; with a rapid disease progression and high early mortality.

While brain biopsy is the gold standard test for diagnosis, it is often unnecessary. A typical clinical presentation with corroborating findings on magnetic resonance imaging, electroencephalography, and cerebrospinal fluid are in most cases sufficient to exclude other causes and establish CJD as the probable diagnosis.

Keywords: Creutzfeldt-Jakob. Prion diseases. Dementia
\end{abstract}

buen nivel de consciencia, fascies inexpresiva y paciente apático aunque colaborador, el lenguaje estaba bien organizado aunque lentificado, estaba desorientado en tiempo y con alteración de la memoria inmediata. No presentaba alteración de músculos óculomotores ni otros nervios craneales y destacaba nistagmos horizontal en ambas miradas laterales. Además, presentaba dismetría y temblor distal en extremidades superiores, con asterixis marcada en manos, discreta hiperreflexia generalizada y marcha inestable con aumento de la base de sustentación.

Los estudios analíticos, que incluyeron función renal, función hepática, iones, función tiroidea, niveles de vitamina B12, no mostraban alteraciones. Los marcadores tumorales, anticuerpos antitiroideos (ATPO) y anticuerpos onconeuronales se encontraban dentro del rango de la normalidad, así como los niveles de cobre y ceruloplasmina. Se realizaron una tomografía computerizada craneal que no mostraba alteraciones, y una resonancia magnética craneal que mostró escasos focos de gliosis isquémica en sustancia blanca en ambos hemisferios cerebrales, sin datos de atrofia parenquimatosa significativa. Se realizaron dos electroencefalogramas que mostraron un trazado deficientemente organizado, asimétrico, muy lentificado sobre todo en región frontemporal derecha, sin datos de actividad paroxística al momento de su realización, compatibles con encefalopatía severa. El estudio serológico de sífilis y VIH fue negativo. Se realizó estudio de líquido cefalorraquídeo, que mostró niveles de glucosa normales, ausencia de leucocitos y discreto aumento de proteinorraquia ( $98 \mathrm{mg} / \mathrm{dL})$, con estudio citológico sin evidencia de malignidad, destacando unos niveles de Proteína 143-3 de $>32,000 \mathrm{AU} / \mathrm{mL}$.

Durante su estancia, el paciente presentó deterioro progresivo del estado general con incapacidad para la ingesta oral, mutismo absoluto, probable ceguera, y el temblor distal en extremidades evolucionó a movimientos mioclónicos, lo que se controló parcialmente con medicación sintomática. Finalmente, falleció tras escasas semanas tras el diagnóstico. 
Dados los hallazgos, la evolución clínica y los datos analíticos, se diagnosticó de probable enfermedad de Creutzfeldt-Jakob.

El examen neuropatológico mostró un tejido con aspecto macroscópico sin alteraciones excepto ligera atrofia en la región posterior occípito-parietal con dilatación de las astas occipitales de los ventrículos laterales. El estudio microscópico reveló como patología fundamental la espongiosis, pérdida neuronal y gliosis extensa en todas las zonas examinadas de la corteza cerebral y localización transcortical, incluyendo la corteza hipocampal. También se observó espongiosis a nivel de los ganglios basales. En el estudio inmunohistoquímico destacó la presencia de depósitos difusos (sinápticos) de proteína priónica (PrP) de localización transcortical en corteza del cíngulo, córtex frontal, parietal y temporal (donde se observan agregados de proteína perivacuolar) y corteza occipital. También se observaron en hipocampo y en hemisferios y vermis cerebelosos. Tras el estudio patológico se confirmó en diagnóstico de enfermedad de Creutzfeltd-Jacob de forma definitiva.

\section{Discusión}

Las enfermedades priónicas son enfermedades neurodegenerativas que se caracterizan por largos períodos de incubación y un progreso inexorable una vez aparecen los síntomas clínicos.

Se han descrito cinco enfermedades priónicas humanas: enfermedad de Creutzfeldt-Jakob (ECJ), que representa más del 90\%, la variante de la enfermedad de Creutzfeldt-Jakob (vECJ), kuru, el síndrome de Gerstmann-Sträussler-Scheinker (GSS), y el insomnio familiar fatal (FFI).

La gran mayoría de casos de ECJ son esporádicos, mientras que 5 a 15\% se deben a casos familiares y menos del $1 \%$ a ECJ iatrogénica'.

La ECJ esporádica tiene una incidencia de un caso por millón de habitantes por año, con una distribución similar en todo el mundo. La edad promedio del inicio de la enfermedad es de entre 57 y 62 años, sin diferencia entre sexos. No se han identificado factores de riesgo para padecer la forma esporádica de la enfermedad, habiéndose postulado varias hipótesis, tal como familiares diagnosticados de ECJ o historia clínica de psicosis².

Clínicamente se caracteriza por un deterioro cognitivo rápidamente progresivo y mioclonías. En su evolución también se pueden observar manifestaciones extrapiramidales, tales como hipocinesia, afectación del tracto córticoespinal, que da lugar a hiperreflexia e espasticidad, y síntomas psiquiátricos, sobre todo en pacientes jóvenes ${ }^{3}$. La media de tiempo entre el diagnóstico y la muerte es de seis meses ${ }^{4,5}$.
Los criterios diagnósticos de probable ECJ son la presencia de demencia progresiva, más al menos dos características clínicas típicas (mioclonías, alteraciones visuales o cerebelosas, afectación extrapiramidal, mutismo acinético), más electroencefalograma típico y/o proteína 14-3-3 positiva en LCR, cuya especificidad es del 80\%; y/0 alteraciones características en la resonancia magnética nuclear cerebral (hiperseñal en núcleo caudado y putamen), habiendo descartado otras entidades con clínica similar ${ }^{6,7,8,9}$.

Sin embargo, el diagnóstico definitivo se confirmará con el estudio anátomo-patológico cerebral, que muestra pérdida neuronal, gliosis, degeneración espongiforme y placas de depósito de proteína priónica; así como con la detección de mutaciones genéticas específicas en caso de la ECJ familiar. Por ello, es de especial importancia la realización de necropsia en pacientes, sobre todo de mediana edad, con deterioro cognitivo cuyo diagnóstico etiológico no se ha esclarecido con las pruebas complementarias realizadas antes de su fallecimiento.

La demencia es una patología frecuente de consulta médica. El interés de este caso radica en la importancia de identificar esta entidad como causa irreversible de deterioro cognitivo en pacientes, habitualmente jóvenes, para proporcionar una atención médica precoz y adecuada, y así optimizar el cuidado y de confort del paciente y su familia, especialmente en las fases finales de la enfermedad.

\section{Bibliografía}

1. Masters CL, Harris JO, Gajdusek DC, et al. Creutzfeldt-Jakob disease: patterns of worldwide occurrence and the significance of familial and sporadic clustering. Ann Neurol 1979; 5:177.

2. Harries-Jones R, Knight R, Will RG, et al. Creutzfeldt-Jakob disease in England and Wales, 1980-1984: a case-control study of potential risk factors. J Neurol Neurosurg Psychiatry 1988; 51:1113

3. Boesenberg C, Schulz-Schaeffer WJ, Meissner B, et al. Clinical course in young patients with sporadic Creutzfeldt-Jakob disease. Ann Neurol 2005; 58:533.

4. Appleby BS, Appleby KK, Crain BJ, et al. Characteristics of established and proposed sporadic Creutzfeldt-Jakob disease variants. Arch Neurol 2009; 66:208.

5. Josephs KA, Ahlskog JE, Parisi JE, et al. Rapidly progressive neurodegenerative dementias. Arch Neurol 2009; 66:201.

6. Kretzschmar HA, Ironside JW, DeArmond SJ, Tateishi J. Diagnostic criteria for sporadic Creutzfeldt-Jakob disease. Arch Neurol 1996; 53:913.

7. Schröter $A, Z$ Zerr I, Henkel $K$, et al. Magnetic resonance imaging in the clinical diagnosis of Creutzfeldt-Jakob disease. Arch Neurol 2000; 57:1751.

8. Hsich G, Kenney K, Gibbs CJ, et al. The 14-3-3 brain protein in cerebrospinal fluid as a marker for transmissible spongiform encephalopathies. N Engl J Med 1996; 335:924.

9. Chohan G, Pennington C, Mackenzie JM, et al. The role of cerebrospinal fluid 14-3-3 and other proteins in the diagnosis of sporadic Creutzfeldt-Jakob disease in the UK: a 10-year review. J Neurol Neurosurg Psychiatry 2010; 81:1243. 